\title{
ANÁLISE FINANCEIRA DE SISTEMAS DE PRODUÇÃO INTEGRADOS NO NORDESTE DO PARÁ
}

\author{
Maria de Nazaré Caetana Marques; ; Rosana Quaresma Maneschy ${ }^{2}$; Jaqueline Fontel de \\ Queiroz ${ }^{3}$; Tahnity Haarad Moura Chaves ${ }^{4}$. \\ ${ }^{1}$ Graduanda em Ciências Econômicas, Universidade Federal do Pará (UFPA), Belém, Pará, Brasil, \\ caetana.maria@yahoo.com.br \\ 2 PPGEDAM/Núcleo de Meio Ambiente (NUMA), UFPA, Belém, Pará, Brasil, romaneschy@ufpa.br \\ ${ }^{3}$ Bolsista PIBIC, UFPA, Belém, Pará, Brasil, jaqueline.fontel07@gmail.com \\ ${ }^{4}$ Mestranda do PPGEDAM/NUMA/UFPA, Belém, Pará, Brasil, tahnityhaarad@gmail.com
}

RESUMO: Sistemas de produção integrados (SPIS) são amplamente utilizados por agricultores familiares no Bioma Amazônia. O objetivo desse trabalho foi modelar SPIs (ex: Consórcios Agrícolas, Sistemas Agroflorestais - SAFs e Sistemas Agrossilvipastoris) no nordeste paraense a fim de subsidiar com informações a geração de políticas públicas de crédito adequadas a esses sistemas praticados pela agricultura familiar. Foi selecionada uma experiência exitosa de SAF acompanhada pela Embrapa Amazônia Oriental no projeto "Transição produtiva e serviços ambientais" para a análise financeira e comparado a sistemas monoculturais. Foram utilizadas com diferentes taxas de juros, a saber: 2,5\% a.a; 7.5\% a.a. e 8,75\% a.a. para as análises de acordo com as linhas, PRONAF-Floresta, Programa Nacional de Apoio ao Médio Produtor Rural - PRONAMP e com o Plano Agrícola e Pecuário 2015/2016 para agricultura empresarial, respectivamente. Os indicadores financeiros utilizados foram: Valor presente líquido, Taxa interna de retorno, Relação benefício custo, Valor anual equivalente e Payback. De acordo com o panorama da viabilidade econômica, - SPI se mostrou eficiente em todas as simulações, porem devido a taxa de juros do PRONAF floresta ser menor (2,5 \% a.a) o retorno econômico foi maior do que nas simulações com as demais taxas.

PALAVRAS-CHAVE: Sistemas de produção integrados; agricultura familiar; análise financeira.

\section{FINANCIAL ANALYSIS OF PRODUCTION SYSTEMS INTEGRATED IN THE NORTHEAST OF PARÁ}

ABSTRACT: Integrated production systems (IPS's) are widely used by family farmers in the Amazon Biome. The objective of this study was to model SPIs (ex: Consortia Agriculture, Agroforestry systems (AFS's) and Agrossilvipastoris Systems) in northeastern Pará to subsidize with information the generation of public policies of appropriate credit to these systems practiced by the family farming. Was selected a successful experience of FAS accompanied by Embrapa Amazônia Oriental in the project "Transition productive and environmental services" for financial analysis and compared to systems monoculturais. Were used with different rates of interest, namely: 2.5\%; 7.5\% per year and $8.75 \%$ p.a. for the analyzes in accordance with the lines, PRONAF-Forest, National Program of Support to the 
Middle Rural Producer - PRONAMP and with the Agricultural and Livestock for 2015/2016 corporate farming, respectively. The financial indicators used were: net present value, internal rate of return, the benefit cost, Equivalent Annual Value and Payback. According to the panorama of economic viability, the SPI was efficient in all simulations, but because the interest rate of the PRONAF forest be lower (2.5\% p.a.) the economic return was greater than in the simulations with the other fees.

KEYWORDS: Integrated production systems; family agriculture; financial analysis.

\section{ANÁLISIS FINANCIERO DE SISTEMAS DE PRODUCCIÓN INTEGRADOS EN EL NORDESTE DEL PARÁ}

RESUMEN: Sistemas de producción integrados (SPIS) son ampliamente utilizados por agricultores familiares en el Bioma Amazonia. El objetivo de este trabajo fue modelar SPIS (por ejemplo: Consorcios Agrícolas, Sistemas Agroforestales - SAFs y Sistemas Agrosilvipastoris) en el nordeste paraense a fin de subsidiar con informaciones la generación de políticas públicas de crédito adecuadas a esos sistemas practicados por la agricultura familiar. Se seleccionó una experiencia exitosa de SAF acompañada por la Embrapa Amazonia Oriental en el proyecto "Transición productiva y servicios ambientales" para el análisis financiero y comparado a sistemas monocultura. Se utilizaron con diferentes tipos de interés, a saber: 2,5\% a.a; 7.5\% a.a. y el 8,75\% a.a. para los análisis de acuerdo con las líneas, PRONAF-Bosque, Programa Nacional de Apoyo al medio Productor Rural PRONAMP y con el Plan Agrícola y Pecuario 2015/2016 para agricultura empresarial, respectivamente. Los indicadores financieros utilizados fueron: Valor presente neto, Tasa interna de retorno, Relación beneficio costo, Valor anual equivalente y Payback. De acuerdo con el panorama de la viabilidad económica, el SPI se mostró eficiente en todas las simulaciones, pero debido a la tasa de interés del PRONAF bosque ser menor (2,5\% aa) el retorno económico fue mayor que en las simulaciones con las demás tasas.

PALABRAS CLAVE: Sistemas de producción integrados, agricultura familiar; Analisis financiero.

A agricultura familiar possui grande representatividade no cenário econômico brasileiro, devido ao fato de que cerca de 85\% dos estabelecimentos agropecuários são formados por propriedades familiares IBGE (2006, apud CARMO et al.,
2016). Dessa forma a degradação ambiental é um grande problema gerado por esse cenário e a transição produtiva vem em busca de solucionar essa problemática através da ampliação da qualidade ambiental de distintos sistemas 
produtivos ao longo do tempo e deve ser utilizada como referencial teórico para orientar o setor agropecuário rumo à sustentabilidade (MATTOS, 2009; BALBINO, 2011).

Nos últimos anos, a discussão sobre transição produtiva passou a influenciar a concepção de políticas agrícolas (Programa ABC no MAPA; linhas especiais do Pronaf no MDA), mas ainda persistem grandes lacunas de informações, instrumentos e metodologias cientificamente validados para tornar estes processos relevantes.

A busca da sustentabilidade tornouse estratégica para promover adaptações às mudanças climáticas globais e superar as atuais e futuras barreiras não tarifárias impostas pelos mercados transnacionais. As barreiras não tarifárias, se por um lado, estimulam processos produtivos com maior sustentabilidade e mais adaptados às mudanças climáticas globais, por outro se notabilizam como uma nova forma de protecionismo dos países desenvolvidos, o que pode comprometer a pauta de comércio exterior de commodities agropecuárias e, consequentemente, o Produto Interno Bruto (PIB) dos países em desenvolvimento. Em especial, no caso brasileiro, vale ressaltar que o setor agropecuário cumpre papel fundamental ao garantir superávit na balança de pagamentos do Estado nacional.

Ribaski e Montoya et al. (2001) apontaram os SAF's como uma alternativa importante para redução da degradação ambiental. Através desses sistemas há um ganho na diversificação produtiva, pois é possível trabalhar com diferentes espécies em uma mesma área, contribuindo para o uso da terra, da mão-de-obra, além de serem excelentes depósitos de gás carbônico.

Na Amazônia destacam-se iniciativas com utilização de sistemas de produção integrados (SIPS) com a utilização de preparo de área sem uso de fogo como o Projetos Tipitamba (KATO et al., 2004) entre outros projetos que não utilizam o fogo. $O$ projeto tem recomendado, ao logo dos anos, a intensificação de sistemas de produção familiares - com base na floresta secundária (capoeira) através do corte-e-trituração mecanizada da capoeira e plantio de leguminosas arbóreas de rápido crescimento para reduzir o pousio. 
Apesar dos benefícios ecológicos que os SIPs apresentam, são poucas informações existentes que possam subsidiar políticas públicas para esses sistemas. Para Hurtienne (2005 p. 21) "o redirecionamento das políticas públicas voltadas para os sistemas de uso da terra na Amazônia é uma tarefa fundamental para qualquer projeto de desenvolvimento sustentável na região", principalmente na questão dos desenhos e da valoração dos mesmos.

De forma que se configuram como um importante problema de pesquisa, as lacunas de informações sobre a viabilidade financeira, instrumentos econômicos e metodologias para apoiar políticas públicas de incentivo a esses sistemas adaptados à agricultura familiar (ex: Consórcios Agrícolas, SAFs e Sistemas Agrossilvipastoris).

Demonstrar a atratividade financeira dos SPIs, através de financiamentos com taxas de juros mais baixas e diferenciadas pode incentivar a disposição dos produtores para adotar os SPIs.

Dessa forme o objetivo geral do trabalho foi modelar um SPIs no nordeste paraense a fim de subsidiar com informações a geração de políticas públicas adequadas a esses sistemas praticados pela agricultura familiar. E mais especificamente: Realizar caracterização socioeconômica e ambiental dos SPIs no nordeste paraense; e Realizar análise financeira dos SPIs para torná-los elegíveis em instrumentos econômicos.

Este estudo foi uma ação do projeto "Transição Produtiva e Serviços Ambientais - FASE I" desenvolvido pela Embrapa, sendo liderado pela Embrapa Cerrados. No Bioma Amazônia a Embrapa Amazônia Oriental é a responsável e tem como entidades parceiras locais: Associação de Desenvolvimento Comunitário de Nova Olinda (ASDCONO), Cooperativa Agrícola Mista de Tomé-Açu (CAMTA), Associação de Produtores e Produtoras Familiar do Município de Tomé-Açu (APPRAFAMTA), órgãos governamentais e outras associações de produtores. A Universidade Federal do Pará e a Universidade Federal Rural da Amazônia participam apoiando as atividades de pesquisa a campo.

projeto define como SPIs aqueles que integram atividades agrícolas, 
pecuárias e florestais numa área produtiva comum, em cultivo consorciado, em sucessão ou rotacionado, com efeitos sinérgicos entre as estruturas agroecossistêmicas (BALBINO et al., 2011).

Foi selecionada uma experiência exitosa de SPI acompanhada pela Embrapa Amazônia Oriental para a análise financeira, sendo comparada a sistemas monoculturais. As análises seguirão as metodologias propostas Arco-Verde (2008) utilizando-se a "Planilha para análise financeira AMAZONSAF" desenvolvida por ArcoVerde e Amaro (2011).

O município de Tomé-Açu pertence à Mesorregião Nordeste Paraense e à Microrregião Tomé-Açu (Figura 1), possui uma população de aproximadamente 60.456 mil habitantes, de acordo com dados do IBGE (2015), sua economia tem forte base nas práticas agropecuárias, possuindo um PIB de aproximadamente $\mathrm{R} \$ 343.622,141 \mathrm{mil}$.

A agricultura é uma das mais importantes fontes de geração de trabalho e renda para a população do município de Tomé-Açu, dado principalmente, a sua significativa participação no valor bruto da produção (62\% do setor agropecuário). É importante destacar que outras atividades econômicas também têm desempenho positivo, a exemplo da extração e beneficiamento de madeira. Entretanto, a atividade agrícola situa-se como base de sustentação sócio-economia do município.

Outra característica importante do município é a diversificação da propriedade, o que torna Tome- Açú como um verdadeiro laboratório aos estudiosos na temática dos sistemas agroflorestais e, consequentemente, na interação biológica entre plantas da mesma e/ou espécies diferentes.

Em Tomé-Açu, concentra-se a colônia mais antiga de japoneses no estado do Pará. A primeira atividade dos imigrantes japoneses foi a horticultura seguida pelo plantio de pimenta-do-reino (Piper nigrun L). Entretanto, a fusariose, doença causada pelo fungo Fusarium solani, provocou redução expressiva dos pimentais do município. Buscando alternativas para minimizar as perdas econômicas provocadas pela fusariose, os produtores optaram por diversificar sua 
produção introduzindo os sistemas agroflorestais. Os sistemas agroflorestais mais exitosos encontrados na Amazônia estão localizados no município de ToméAçu (REGO, 2015).

O município de Tomé-Açú, de acordo com boletim agropecuário do Estado do Pá 2015, é o oitavo município paraense com maior de produção agrícola, o que representa 2,83\% da produção total do Estado, onde há destaque para ocupação de culturas perenes como: pimenta-doreino, cacau, dendê, cupuaçu, manga, castanha-do-brasil, acerola, essências florestais, pastagens entre outras. (FAPESPA, 2015)

Figura 1. Mapa de localização de Tomé-Açu, Pará.
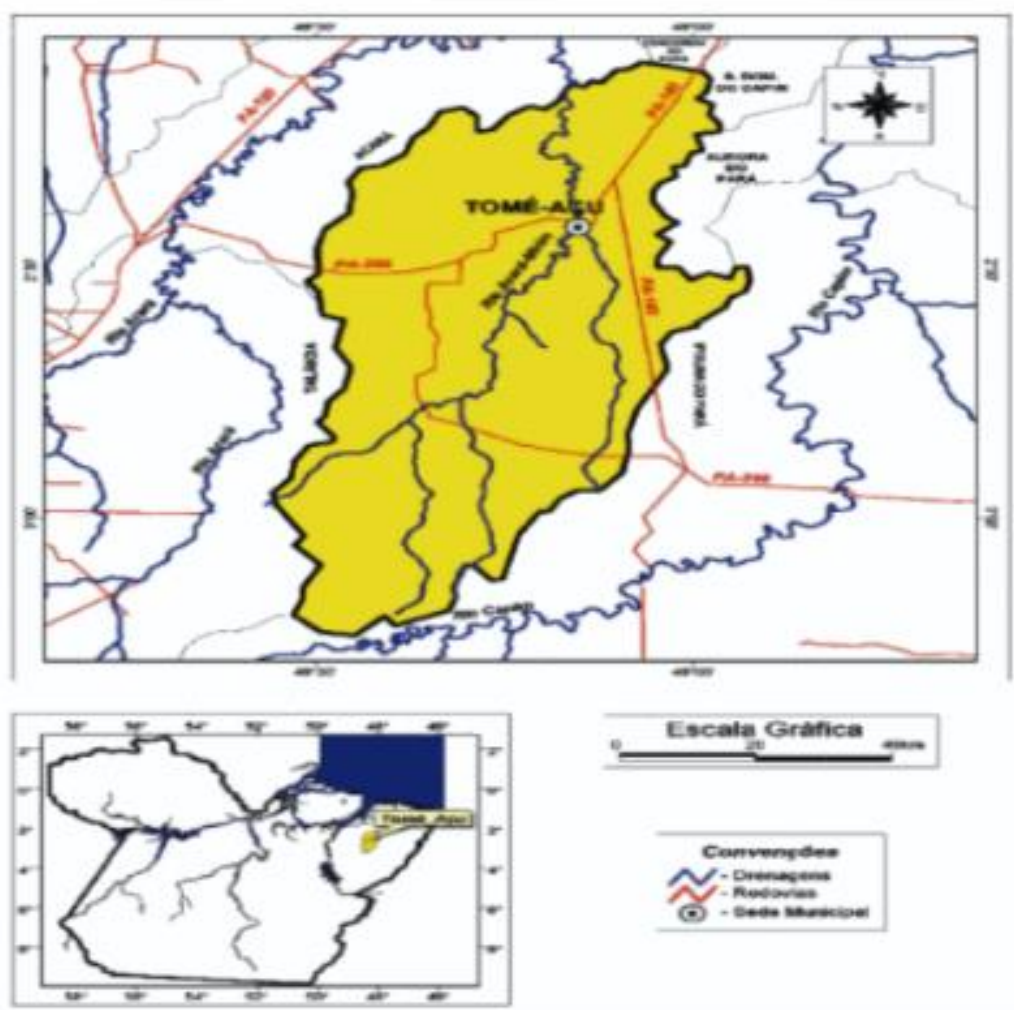

Escala Grafica

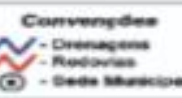

Fonte: Antônio G. S. Campos apud Boletim Agrometeorológico, Tomé-Açu, PA (2008).

As análises seguiram a metodologia proposta por Arco-Verde e Amaro (2011) utilizando-se a "Planilha para análise financeira AMAZONSAF" desenvolvida pelos autores. Foram analisados os seguintes indicadores financeiros: Valor Presente Líquido (VPL), Relação BenefícioCusto (B/C), Taxa Interna de Retorno (TIR), 
Tempo de Retorno do Investimento (TRI), Valor Atual dos Custos (VAC) e Valor Atual das Receitas (VAR).

Para análise de rentabilidade foram considerados os fluxos de caixa de entrada (receitas ou benefícios) e saída (custos), que ocorrerão em um horizonte definido de tempo, de 20 anos.

Valor Presente Líquido (VPL) apresenta os valores líquidos no instante considerado inicial, a partir de um fluxo de caixa formado por uma série de receitas e custos.

$V P L=\sum_{j=0}^{n} R_{J}(1+i)^{-j}-\sum_{j=0}^{n} R_{J}(1+i)^{-j}$

Onde: $\mathrm{Rj}$ = receitas no final do ano ou do período de tempo j considerado; $\mathrm{Cj}=$ custos no final do ano ou do período de tempo $\mathrm{j}$ considerado; $\mathrm{n}=$ duração do projeto em anos ou em número de períodos de tempo; $\mathrm{i}=$ taxa anual de juros, expressa em porcentagem, considerada de $12 \%$ a.a.

A Relação Benefício Custo (B/C) divide os benefícios atualizados pelos custos atualizados indicando quanto os benefícios superam ou não os custos totais (ARCO-VERDE, 2008).

$$
\mathrm{R}_{\mathrm{b} / \mathrm{c}}=\frac{\sum_{\mathrm{t}=0}^{\mathrm{n}} \mathrm{R}_{\mathrm{j}}(1+\mathrm{i})^{-\mathrm{j}}}{\sum_{\mathrm{t}=0}^{\mathrm{n}} \mathrm{C}_{\mathrm{j}}(1+\mathrm{i})^{-\mathrm{j}}}
$$

Onde: $\mathrm{Rj}=$ receitas no final do ano ou do período de tempo $\mathrm{j}$ considerado; $\mathrm{Cj}$ = custos no final do ano ou do período de tempo $\mathrm{j}$ considerado; $\mathrm{n}=$ duração do projeto em anos ou em número de períodos de tempo; $\mathrm{i}$ = taxa anual de juros, expressa na forma unitária.

A Taxa Interna de Retorno (TIR) é a taxa de desconto que iguala o valor presente dos ingressos ao valor presente dos custos, ou seja, iguala o VPL a zero. Também pode ser entendida como a taxa percentual do retorno do capital investido. De acordo com Diogo et. al. (2017) a viabilidade do projeto também pode ser avaliada pela taxa interna de retorno (TIR), A TIR é uma demonstração da rentabilidade do projeto.

$$
0=\sum_{\mathrm{t}=0}^{\mathrm{n}} \frac{\mathrm{R}_{\mathrm{t}}-\mathrm{C}_{\mathrm{t}}}{\left(1+\mathrm{i}^{*}\right)^{\mathrm{t}}}
$$

Onde: $i^{\star}=$ Taxa interna de retorno; $\mathrm{Bt}=$ receita total ao final do ano ou período de tempo; $\mathrm{Ct}=$ custo total ao final do ano ou período de tempo; $\mathrm{n}=$ duração do projeto em anos ou em número de períodos de tempo; $j$ = duração do período do projeto, em anos ou período de tempo.

O Tempo de Retorno do Investimento (TRI) consiste em determinar quanto tempo de funcionamento será necessário para que o projeto permita aos investidores o retorno do capital investido. 
O TRI equivale ao inverso da rentabilidade simples.

$$
\text { TRI }=1 / R
$$

Onde: TRI = tempo de retorno do investimento; $\mathrm{R}$ = rentabilidade do investimento.

O Valor Atual dos Custos (VAC): consiste em determinar o valor atualizado dos custos, de acordo com a taxa de juros utilizada, durante um determinado período de avaliação (ARCO- VERDE, 2008).

$$
V A C=\left[C_{1} /(1+i)^{n}\right.
$$

Onde: $\mathrm{Cl}$ = valor atual dos custos; $\mathrm{i}=$ taxa de juros; $\mathrm{n}$ = período em que os ingressos ou os custos ocorreram.

As informações utilizadas para a simulação nos modelos propostos para a análise financeira são oriundas do banco de dados do Projeto: Transição Produtiva e Serviços Ambientais - FASE I, a partir do trabalho de campo de Rego (2015).

Foram selecionadas as seguintes densidades/espécies para o sistema analisado: 2500 unid/ha de pimenta do reino (espaçamento de $2 \times 2 \mathrm{~m}$ ), 1111 unid/ha de cacau (espaçamento de $3 \times 3$ m), 100 unid/ha de Mogno (espaçamento de $10 \times 10$ m), 125000 unid/ha arroz (espaçamento 0,4×0,2m) plantados nas linhas do Mogno, e 400 unid/ha de açaí (espaçamento de $5 \times 5 \mathrm{~m}$ ).

Na literatura pertinente buscaram-se informações quanto a tratos culturais necessários, períodos de desbastes das espécies arbóreas e estimativa de produção (Quadro1).

Quadro 1. Referenciais técnicos das espécies utilizadas nos modelos propostos na simulação

\begin{tabular}{|c|c|c|}
\hline ESPÉCIES & DENSIDADE DE PLANTIO/RECOMENDAÇÃO & REFERÊNCIA \\
\hline Mandioca & $\begin{array}{l}\text { 10.000 plantas/ha (espaçamento de } 1,0 \text { × 1,0m). } \\
\text { Plantio, capina, manutenção e colheita. }\end{array}$ & $\begin{array}{c}\text { Castro (2014) apud } \\
\text { Mattos e Cardoso (2003) }\end{array}$ \\
\hline Arroz & 125000 plantas/ha (espaçamento 0,4×0,2 m) & Rego (2015) \\
\hline Pimenta do Reino & 2500 plantas/ha (espaçamento 2× 2 m) & Rego (2015) \\
\hline Açaí & 400 plantas/ha (espaçamento $5 \times 5$ m) & Rego (2015) \\
\hline Cacau & 1.111 plantas/ha (espaçamento $3 \times 3$ m) & $\begin{array}{c}\text { Castro (2014) apud Silva } \\
\text { Neto et al. (2001) }\end{array}$ \\
\hline Mogno & 100 plantas/ha (espaçamento 10 × 10m) & Rego (2015) \\
\hline
\end{tabular}
econômica. 
Utilizou-se as taxas de 2,5\% a.a.; 7.5\% a.a. e 8,75\% a.a. para as análises de acordo com o Programa Nacional de Fortalecimento da Agricultura Familiar (PRONAF) linha Floresta, Programa Nacional de Apoio ao Médio Produtor Rural (PRONAMP) e do Plano Agrícola e Pecuário
2015/2016 para agricultura empresarial, respectivamente.

No Quadro 2 estão dispostos os modelos de sistemas de produção utilizados na simulação financeira. Os preços de insumos e venda de produtos que serão utilizados na análise financeira (Tabela 1).

Quadro 2. Modelos propostos para a análise financeira.

\begin{tabular}{|l|l|}
\hline Modelo & Componentes \\
\hline \multirow{5}{*}{ SAF } & Arroz/Mandioca (Cultura anual) \\
\cline { 2 - 2 } & Mogno (Plantio florestal) \\
\cline { 2 - 2 } & Cacau e Açaí (Perene) \\
\cline { 2 - 2 } & Pimenta - do- reino (Semi-perene) \\
\cline { 2 - 2 } & Arroz/Mandioca x Mogno x Cacau x Açaí x Pimenta do reino \\
\hline
\end{tabular}

O SAF analisado apresentou distribuição de mão de obra ao longo dos 20 anos da simulação (Figura 2). O custo da mão-de-obra no SAF é distribuído em função das demandas periódicas realização de coroamento e limpeza da área, sobretudo para diminuição da competição entre espécies invasoras e a espécie florestal, bem como o corte e transporte da madeira ao final do ciclo.

Verificou-se VPL positivo e TIR superior às taxas descontos simuladas 2,5\% a.a. 7.5\% a.a. e 8,75\% a.a. De acordo com o panorama da viabilidade econômica, o SAF se mostrou eficiente em todas as simulações, porem devido a taxa de juros do PRONAF floresta ser menor (2,5\% a.a) o retorno econômico é maior do que nas simulações com as demais taxas (Tabela 2).

Ao simularmos o SAF utilizando as diferentes taxas de juros de 2,5\% a.a. 7.5\% a.a. e 8,75\% a.a, verificou-se que em todos as simulações o SAF apresentou viabilidade economicamente significativa, haja vista que a $\mathbf{R}_{\mathbf{b} / \mathbf{c}}$ se mantem com valores próximos em todas as simulações; e comprovou que as receitas foram maiores que os custos descontados com todas as taxas de juros simuladas no sistema analisado, pois a cada $R \$ 1,00$ investido existe o retorno de tais valores líquidos (SANTANA, 2005). 
Tabela 1. Parâmetros utilizados na simulação dos modelos.

\begin{tabular}{|c|c|c|c|}
\hline Parâmetros & Especificação & Unidade & Valor $(R \$)$ \\
\hline Gerais & Hora Trator (com tratorista) & Hora Diária & 140,00 \\
\hline Preço de Venda dos & Pimenta-do-Reino & $\mathrm{Kg}$ & 28,00 \\
\hline \multirow{5}{*}{ Produtos } & Mogno & $m^{3}$ & 250,00 \\
\hline & Cacau & $\mathrm{Kg}$ & 7,00 \\
\hline & Açaí & $\mathrm{Kg}$ & 2,43 \\
\hline & Arroz & $\mathrm{Kg}$ & 0,80 \\
\hline & Mandioca & $\mathrm{Kg}$ & 0,25 \\
\hline Insumos: Plantio de & Mudas & unid & 3,50 \\
\hline \multirow[t]{10}{*}{ Pimenta-do-Reino } & NPK 08-20-20 & $\mathrm{kg}$ & 3,10 \\
\hline & Arad & $\mathrm{kg}$ & 1,32 \\
\hline & Farinha de osso & $t$ & 1090,00 \\
\hline & Torna de mamona & $\mathrm{t}$ & $1.110,00$ \\
\hline & Herbicida (glifosato) & I & 25,60 \\
\hline & Inseticida (decis 25CE) & । & 84,20 \\
\hline & Estacas de madeira & unid & 8,00 \\
\hline & Óleo mineral & । & 16,60 \\
\hline & FTE BR 12 & $\mathrm{~kg}$ & 60,00 \\
\hline & Cloreto de potássio & $\mathrm{kg}$ & 2,20 \\
\hline \multirow{8}{*}{$\begin{array}{l}\text { Insumos: Plantio de } \\
\text { Mogno }\end{array}$} & Adubo Orgânico & $\mathrm{kg}$ & 0,40 \\
\hline & NPK 10-26-26 & $\mathrm{kg}$ & 0,74 \\
\hline & Mudas & unidade & 4,00 \\
\hline & Superfosfato & $\mathrm{kg}$ & 2,00 \\
\hline & Herbicida & | & 20,00 \\
\hline & Formicida & I & 9,15 \\
\hline & Inseticida & I & 47,00 \\
\hline & FTE & $\mathrm{kg}$ & 0,40 \\
\hline \multirow{7}{*}{$\begin{array}{l}\text { Insumos: Plantio de } \\
\text { Cacau }\end{array}$} & Mudas & Unidade & 4,50 \\
\hline & Calcário & $\mathrm{kg}$ & 0,20 \\
\hline & N & $\mathrm{kg}$ & 3,94 \\
\hline & $\mathrm{P}$ & $\mathrm{kg}$ & 3,91 \\
\hline & K & $\mathrm{kg}$ & 2,34 \\
\hline & Herbicida & l & 19,42 \\
\hline & Inseticida & । & 47,00 \\
\hline \multirow{6}{*}{$\begin{array}{l}\text { Insumos: Plantio de } \\
\text { Açaí }\end{array}$} & Mudas & unid & 1,50 \\
\hline & NPK 08-20-20 & $\mathrm{kg}$ & 3,10 \\
\hline & Arad & $\mathrm{kg}$ & 1,32 \\
\hline & Cloreto de Potássio & $\mathrm{kg}$ & 2,20 \\
\hline & Irrigação (poço, tubulação, equipamentos) & unid & $10.000,00$ \\
\hline & Manutenção da irrigação & unid & $2.500,00$ \\
\hline \multirow{9}{*}{$\begin{array}{l}\text { Insumos: Plantio de } \\
\text { Arroz } \\
\text { Insumos: Plantio de } \\
\text { Mandioca }\end{array}$} & Sementes & $\mathrm{kg}$ & 2,50 \\
\hline & NPK 10-28-20 & $\mathrm{kg}$ & 2,45 \\
\hline & Manivas (20 cm) & m3 & 20,00 \\
\hline & Nitrogênio & $\mathrm{kg}$ & 3,94 \\
\hline & Fósforo & $\mathrm{kg}$ & 3,91 \\
\hline & Cloreto de Potássio & $\mathrm{kg}$ & 2,34 \\
\hline & Formicida & l & 0,44 \\
\hline & Herbicida & I & 17,00 \\
\hline & Inseticida & I & 19,42 \\
\hline
\end{tabular}

Fonte: Adaptado de Rego (2015). 
Figura 2. Demanda de mão-de-obra do SAF simulado.

Demanda Total de Mão-de-Obra

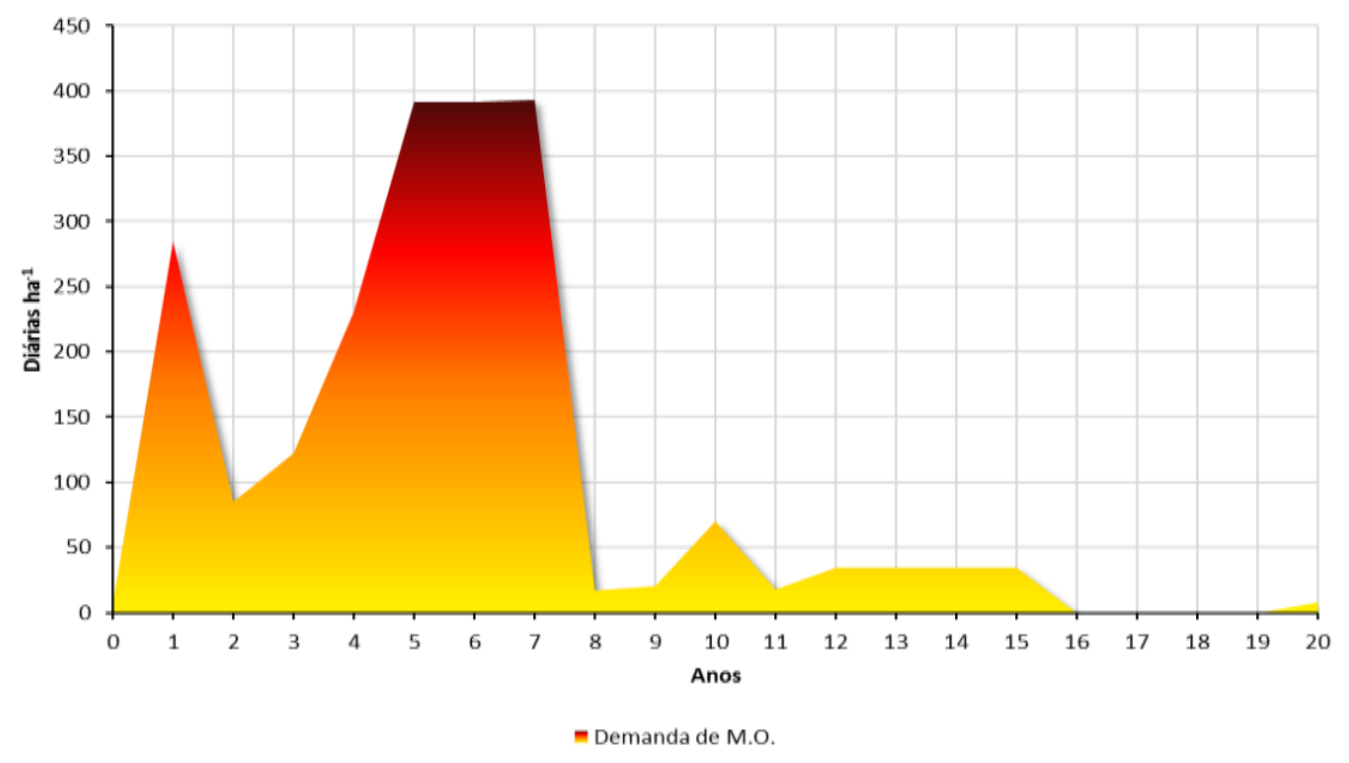

Fonte: Autor Próprio

Tabela 2. Valor presente líquido (VPL), Taxa interna de retorno (TIR), Relação benefício custo $\left(\boldsymbol{R}_{\boldsymbol{b} / \boldsymbol{c}}\right)$ e valor anual equivalente (VAE) nos modelos simulados.

\begin{tabular}{lcccccc}
\hline MODELO & $\begin{array}{c}\text { Taxa de juros } \\
\text { (\% aa) }\end{array}$ & VPL (R\$) & TIR (\%) & $\boldsymbol{R}_{\boldsymbol{b} / \boldsymbol{c}}(\mathrm{R} \$)$ & VAE (R\$) & $\begin{array}{c}\text { Payback } \\
\text { (Anos) }\end{array}$ \\
\hline SAF & $2,5 \%$ & $670.952,41$ & $101,45 \%$ & 4,2 & $43.039,67$ & 3 \\
& $7,5 \%$ & $484.503,27$ & $101,45 \%$ & 3,7 & $47.525,99$ & 4 \\
& $8,75 \%$ & $456.149,78$ & $101,45 \%$ & 3,8 & $49.082,52$ & 4 \\
\hline
\end{tabular}

Fonte: Autor Próprio.

De acordo com Cosenza e Neto et. al. (2017), "a viabilidade do projeto também pode ser avaliada pela taxa interna de retorno (TIR), que seria a taxa anual que retorna o capital investido". No caso das simulações apresentadas, houve um retorno de 1,45\%, o que mostra a rentabilidade positiva dos sistemas, independente da taxa de juros aplicada.
Na análise econômica do SAF com diferentes taxas de juros, foram examinados os custos e benefícios em função dos preços de mercado, determinando sua relação com os indicadores econômicos, possibilitando verificar a viabilidade do projeto.

Um dos principais benefícios gerados pelos SAFs, do ponto de vista dos agricultores familiares, é a produção de 
alimentos voltados para própria família, além da elevação da renda e uma expressiva diversificação na produção, porém há certos cuidados a serem tomados na implantação dos sistemas, um deles é a forma como as espécies são arranjadas, pois é através desse arranjo que depende a rentabilidade do sistema, tem haver o cuidado em implantar modelos de acordo com a região de estudo, utilizando a tecnologia adequada e visando a demando do mercado local.

E na comparação econômica feita nesta pesquisa foi verificado que o ganho econômico produzido na implantação de um SAF é verídico mesmo utilizando diferentes taxas de juros, que há de fato a elevação da renda do produtor.

\section{REFERÊNCIAS}

ARCO-VERDE, M. F. Sustentabilidade Biofísica e Socioeconômica de sistemas Agroflorestais na Amazônia brasileira 2008. 188 p. Tese (Doutorado em Ciências Florestais) - Universidade Federal do Paraná, Curitiba.. 2008. Disponível em: http://www.floresta.ufpr.br/posgraduacao/defesas/pdf_dr/2008/t242_02 84-D.pdf. Acesso em: 11/12/2016.

ARCO-VERDE, M. F.; AMARO, G. Cálculo de Indicadores Financeiros para Sistemas Agroflorestais. Boa Vista, RR. Documentos / Embrapa Roraima, v.44. n. 1- 48p. 2011.
CARMO, J. M.; BAUTE, G.; BAUTE, T.; IWAMOTO, V. T. Proposta de aplicativo para pequeno produtor: consorciamento de culturas em sistemas agroflorestais. Revista Eletrônica Competências Digitais para Agricultura Familiar.v.1, n. 2, p.50-61, 2016.

COSENZA D. N.; OLIVEIRA NETO, S. N.; JACOVINE, L. A. G.; RODRIGUES, C. R.; RODE, R.; SOARES, V. P.; LEITE, H. G. Avaliação econômica de projetos de sistemas agroflorestais. Pesquisa Florestal Brasileira, v. 36, n. 88, p. 527-536, 2017.

FAPESPA-FUNDAÇÃO AMAZÔNIA DE AMPARO A ESTUDOS E PESQUISAS DO PARÁ. Boletim Agropecuário Sustentabilidade Biofísica e Socioeconômica de Sistemas Agroflorestais na Amazônia Brasileira. do Estado do Pará 2015. Belém, no 1, julho. 2015.

HURTIENNE, T. Agricultura familiar e desenvolvimento rural sustentável na Amazônia. Novos Cadernos NAEA, v. 8, n. 1 - p. 019-071 jun. 2005.

KATO, O.R.; KATO, M.S.A. SÁ, T.D. de A.; FIGUEIREDO, R. Plantio direto na capoeira. Ciência e Ambiente, v. 29, p. 99-111, 2004.

REGO, A. K. Viabilidade Econômica de Sistemas Agroflorestais Sequenciais no Nordeste Paraense. Dissertação (Mestrado em Ciências Florestais). Instituto de Ciências Agrárias. Universidade Federal Rural do Pará. 2015.

SANTANA, A. C.; TOURINHO, M. M. Notas sobre avaliação sócio-econômica de sistemasagroflorestais na Amazônia. In: AGUIAR, D. R. D.; PINHO, J. B. 
Agronegócio brasileiro: desafios e perspectivas. Brasília: SOBER, 1998. v. 2. p. 165-177.1998.

SILVA, M. M. Projeto Roça Sem Queimar: uma proposta de manejo agroecológico para a região da Transamazônica - Pará. 2003. 179 f. Dissertação (Pós - graduação em Agroecossistemas). Centro de Ciências Agrárias. Universidade Federal de Santa Catarina. Florianópolis.

RIBASKI, J.; MONTOYA, L. J.; RODIGHERI, H. R. Sistemas Agroflorestais: Aspectosambientais e socioeconômicos. Informe Agropecuário, v.22, n.212, p. 6167, 2001. 